\title{
NEW EXTENSIONS OF CLINE'S FORMULA FOR GENERALIZED DRAZIN-RIESZ INVERSES
}

\author{
ABDELAZIZ TAJMOUATI, MOHAMMED KARMOUNI, AND M. B. MOHAMED AHMED
}

\begin{abstract}
In this note, Cline's formula for generalized Drazin-Riesz inverses is proved. We prove that if $A, D \in \mathcal{B}(X, Y)$ and $B, C \in \mathcal{B}(Y, X)$ are such that $A C D=D B D$ and $D B A=A C A$, then $A C$ is generalized Drazin-Riesz invertible if and only if $B D$ is generalized Drazin-Riesz invertible, and that, in such a case, if $S$ is a generalized Drazin-Riesz inverse of $A C$ then $T:=B S^{2} D$ is a generalized Drazin-Riesz inverse of $B D$.
\end{abstract}

\section{INTRODUCTION}

Let $X, Y$ be Banach spaces and let $\mathcal{B}(X, Y)$ denote the algebra of all bounded linear operators from $X$ to $Y$. For $Y=X$ we write $\mathcal{B}(X, X)=\mathcal{B}(X)$. A bounded operator $T \in \mathcal{B}(X)$ is said to be Drazin invertible if there exists a positive integer $k$ and an operator $S \in \mathcal{B}(X)$ such that (see [5]):

$$
S T=T S, \quad S^{2} T=S, \quad \text { and } \quad T^{k+1} S=T^{k} .
$$

The concept of Drazin invertible operators has been generalized by Koliha [7] by replacing the third condition in this definition with the condition that $T S T-T$ is quasi-nilpotent. Recall that a bounded operator $T \in \mathcal{B}(X)$ is Riesz, if $T-\lambda I$ is Fredholm in the usual sense for every $\lambda \in \mathbb{C} \backslash\{0\}$ (see [1]).

Recently, Živković-Zlatanović and Cvetković [14] introduced and studied a new concept of pseudo-inverse to extend the Koliha concept to "generalized DrazinRiesz invertible". In fact, an operator $T \in \mathcal{B}(X)$ is said to be generalized DrazinRiesz invertible, if there exists $S \in \mathcal{B}(X)$ such that

$$
T S=S T, \quad S T S=S, \quad \text { and } T S T-T \text { is Riesz. }
$$

In this case $S$ is called a generalized Drazin-Riesz inverse of $T$. Until now, the uniqueness of Drazin-Riesz's generalized inverse remains an open question. Živković-Zlatanović and Cvetković also showed that $T$ is generalized Drazin-Riesz invertible iff it has a direct sum decomposition $T=T_{1} \oplus T_{0}$ with $T_{1}$ invertible and $T_{0}$ Riesz. The generalized Drazin-Riesz spectrum of $T \in \mathcal{B}(X)$ is defined by

$$
\sigma_{g D R}(T)=\{\lambda \in \mathbb{C}: T-\lambda I \text { is not generalized Drazin-Riesz invertible }\} .
$$


Jacobson's Lemma [2] asserts that if $A, B \in \mathcal{B}(X)$, then

$$
A B-I \text { is invertible } \Longleftrightarrow B A-I \text { is invertible. }
$$

As extensions of Jacobson's lemma, Corach et al. 4] investigated (1.1) under the assumption $A B A=A C A$. They studied common properties of $A C$ and $B A$ from an algebraic viewpoint and also obtained some nice topological analogues ([10] [11] [12]).

For an associative ring $R$ with unit, Cline [3] showed that if $a, b \in R$ such that $a b$ is Drazin invertible then so is $b a$ and in this case the Drazin inverse of $b a$ is $(b a)^{D}=b\left((a b)^{D}\right)^{2} a$. This formula is called Cline's formula. Lately, Cline's formula for Drazin and generalized Drazin inverses in a ring under the conditions $a c d=d b d, d b a=a c a$, was extended respectively by Miller and Zguitti [8] and by Zenga et al. [13. Recently, we gave Cline's formula for generalized Drazin-Riesz inverse under the assumption $A B A=A C A$, where $A, B, C \in \mathcal{B}(X)$, see [6] .

In this note, we propose to give Cline's formula for the generalized Drazin-Riesz inverse for bounded linear operators satisfying the operator equation set

$$
\left\{\begin{array}{l}
A C D=D B D, \\
D B A=A C A,
\end{array}\right.
$$

where $A, D \in \mathcal{B}(X, Y)$ and $B, C \in \mathcal{B}(Y, X)$.

\section{MAin RESULtS}

Lemma 2.1. Let $A, D \in \mathcal{B}(X, Y)$ and $B, C \in \mathcal{B}(Y, X)$ such that $\left\{\begin{array}{l}A C D=D B D, \\ D B A=A C A\end{array}\right.$ Then $A C$ is Riesz if and only if $B D$ is Riesz.

Proof.

$$
\begin{aligned}
A C \text { is Riesz } & \Longleftrightarrow \lambda I-A C \text { is Fredholm for all } \lambda \in \mathbb{C} \backslash\{0\} \\
& \Longleftrightarrow \lambda I-B D \text { is Fredholm for all } \lambda \in \mathbb{C} \backslash\{0\} \\
& \Longleftrightarrow B D \text { is Riesz. }
\end{aligned}
$$

See [9, Theorem 3.1].

Theorem 2.2. Let $A, D \in \mathcal{B}(X, Y)$ and $B, C \in \mathcal{B}(Y, X)$ such that $\left\{\begin{array}{l}A C D=D B D, \\ D B A=A C A\end{array}\right.$ Then, $A C$ is generalized Drazin-Riesz invertible if and only if $B D$ is generalized Drazin-Riesz invertible. In this case if $S$ is a generalized Drazin-Riesz inverse of $A C$ then $T=B S^{2} D$ is a generalized Drazin-Riesz inverse of $B D$.

Proof. $\Rightarrow)$ Suppose that AC is generalized Drazin-Riesz inverse, then there exists $S \in \mathcal{B}(Y)$ such that

$$
\left\{\begin{array}{l}
S(A C)=(A C) S \\
S(A C) S=S \\
(A C) S(A C)-A C \text { is Riesz. }
\end{array}\right.
$$


Let $T=B S^{2} D$, then we obtain

$$
\begin{aligned}
T(B D) & =\left(B S^{2} D\right) B D \\
& =B S^{2}(D B D) \\
& =B S^{2}(A C D) \\
& =B\left(S^{2} A C\right) D \\
& =B S D .
\end{aligned}
$$

On the other hand, we have

$$
\begin{aligned}
(B D) T & =(B D) B S^{2} D \\
& =B D B(S) S D \\
& =B D B\left(A C S^{2}\right) S D \\
& =B(D B A) C S^{3} D \\
& =B(A C A) C S^{3} D \\
& =B\left(A C A C S^{3}\right) D \\
& =B(S) D \\
& =B S D .
\end{aligned}
$$

Hence, we deduce that

$$
T(B D)=(B D) T
$$

Next, also we have

$$
\begin{aligned}
T(B D) T & =B S^{2} D(B D) B S^{2} D \\
& =B S^{2} D B D B(S) S D \\
& =B S^{2} D B D B\left(A C S^{2}\right) S D \\
& =B S^{2}((D B D) B A C) S^{3} D \\
& =B S^{2}((A C D) B A C) S^{3} D \\
& =B S^{2}(A C(D B A) C) S^{3} D \\
& =B S^{2}(A C(A C A) C) S^{3} D \\
& =B S^{2}(A C A C A C) S^{3} D \\
& =B S\left(A C A C A C S^{4}\right) D \\
& =B S(S) D \\
& =B S^{2} D \\
& =T .
\end{aligned}
$$

So

$$
T(B D) T=T
$$


Finally, let $Q=I-A C S$. Since we have $Q A C=(I-A C S) A C=A C-A C S A C$, $Q A C$ is Riesz. Also we have

$$
\begin{aligned}
B D-(B D)^{2} T & =B D-(B D)^{2} T \\
& =B D-(B D)^{2} B S^{2} D \\
& =B D-(B D B D) B(S) S D \\
& =B D-B D B D B\left(A C S^{2}\right) S D \\
& =B D-B D B D B A C S^{3} D \\
& =B D-B D B(D B A) C S^{3} D \\
& =B D-B D B(A C A) C S^{3} D \\
& =B D-B(D B A) C A C S^{3} D \\
& =B D-B(A C A) C A C S^{3} D \\
& =B D-B A C\left(A C A C S^{3}\right) D \\
& =B D-B A C(S) D \\
& =B(I-A C S) D \\
& =B Q D, \\
& =D B(I-A C S) A \\
& =D B A-(D B A) C S A \\
& =A C A-(A C A) C S A \\
& =A C(I-A C S) A \\
& =A C Q A
\end{aligned}
$$

and

$$
\begin{aligned}
D B Q D & =D B(I-A C S) D \\
& =D B A-(D B A) C S D \\
& =A C A-(A C A) C S D \\
& =A C(I-A C S) D \\
& =A C Q D .
\end{aligned}
$$

Then, we deduce that

$$
\left\{\begin{array}{l}
D B Q A C=A C Q A C \\
D B Q D B=A C Q D B
\end{array}\right.
$$

Therefore, by Lemma 2.1, we deduce that $D B Q=B D-(B D)^{2} T$ is Riesz.

In summary, $B D$ is generalized Drazin-Riesz invertible.

$\Leftarrow)$ The other direction is automatic by symmetry of $A$ with $D$ and $C$ with $B$.

As a direct consequence of Theorem 2.2 we have the following corollaries. 
Corollary 2.3. Let $A, D \in \mathcal{B}(X, Y)$ and $B, C \in \mathcal{B}(Y, X)$ such that $\left\{\begin{array}{l}A C D=D B D \\ D B A=A C A\end{array}\right.$ Then,

$$
\sigma_{g D R}(A C)=\sigma_{g D R}(B D)
$$

Corollary 2.4. Let $A \in \mathcal{B}(X, Y)$ and $B \in \mathcal{B}(Y, X)$. Then,

$$
\sigma_{g D R}(A B)=\sigma_{g D R}(B A) \text {. }
$$

If $A=D$ and $B=C$, we have ([ᄄ], Theorem 2.1]):

Corollary 2.5. If $A, B, C \in \mathcal{B}(X)$ satisfy $A B A=A C A$, then

$A C$ is generalized Drazin-Riesz invertible

$$
\Longleftrightarrow B A \text { is generalized Drazin-Riesz invertible. }
$$

In this case if $S$ is a generalized Drazin-Riesz inverse of $A C$ then $T=B S^{2} A$ is a generalized Drazin-Riesz inverse of $B A$.

In the following example, we prove that the results obtained in this work are a proper generalization of the corresponding ones in [6]

Example. Let $S_{1} \in \mathcal{B}(X), S_{2}, T_{1} \in \mathcal{B}(Y, X)$, and $T_{2} \in \mathcal{B}(X, Y)$ satisfying $S_{1}=$ $S_{2} T_{2}$ and $T_{2} T_{1} \neq I$. Let

$$
A=\left(\begin{array}{cc}
0 & S_{2} \\
0 & 0
\end{array}\right), \quad B=\left(\begin{array}{cc}
S_{1} & 0 \\
0 & 0
\end{array}\right), \quad C=D=\left(\begin{array}{cc}
I & T_{1} \\
T_{2} & I
\end{array}\right) .
$$

It is easy to see that $A C B=B C B$ and $B C A=A C A$, but $C A C \neq C B C$. Then Cline's formula for generalized Drazin-Riesz inverses can only be obtained from the results above, but not from the corresponding ones in [6].

We finish this work with the following natural question.

Question: Is Cline's formula for generalized Drazin-Riesz inverses in a ring with identity true?

\section{ACKNOWLEDGements}

We gratefully acknowledge the constructive comments of the referee concerning this paper.

\section{REFERENCES}

[1] P. Aiena, Fredholm and Local Spectral Theory, with Applications to Multipliers, Kluwer, 2004. MR 2070395

[2] B. A. Barnes, Common operator properties of the linear operators RS and SR, Proc. Amer. Math. Soc. 126 (1998), no. 4, 1055-1061. MR 1443814.

[3] R. E. Cline, An application of representation for the generalized inverse of a matrix, MRC Technical Report 592, 1965.

[4] G. Corach, B. Duggal, R. Harte, Extensions of Jacobson's lemma, Comm. Algebra 41 (2013), no. 2, 520-531. MR 3011779

[5] M. P. Drazin, Pseudo-inverses in associative rings and semigroups, Amer. Math. Monthly 65 (1958), 506-514. MR 0098762 
[6] M. Karmouni, A. Tajmouati, A Cline's formula for the generalized Drazin-Riesz inverses, Funct. Anal. Approx. Comput. 10 (2018), no. 1, 35-39. MR 3782829

[7] J. J. Koliha, A generalized Drazin inverse, Glasgow Math. J. 38 (1996), no. 3, 367-381. MR 1417366

[8] V. G. Miller, H. Zguitti, New extensions of Jacobson's lemma and Cline's formula, Rend. Circ. Mat. Palermo (2) 67 (2018), no. 1, 105-114. MR 3777821

[9] K. Yan, X. Fang, Common properties of the operator products in spectral theory, Ann. Funct. Anal. 6 (2015), no. 4, 60-69. MR 3365981.

[10] K. Yan, X. Fang, Common properties of the operator products in local spectral theory, Acta Math. Sin. (Engl. Ser.) 31 (2015), no. 11, 1715-1724. MR 3406670.

[11] Q. P. Zeng, H. J. Zhong, Common properties of bounded linear operators $A C$ and $B A$ : spectral theory, Math. Nachr. 287 (2014), no. 5-6, 717-725. MR 3193947

[12] Q. P. Zeng, H. J. Zhong, New results on common properties of the products $A C$ and $B A$, J. Math. Anal. Appl. 427 (2015), no. 2, 830-840. MR 3323010

[13] Q. Zeng, Z. Wu, Y. Wen, New extensions of Cline's formula for generalized inverses, Filomat 31 (2017), no. 7, 1973-1980. MR 3635233

[14] S. Č. Živković-Zlatanović, M. D. Cvetković, Generalized Kato-Riesz decomposition and generalized Drazin-Riesz invertible operators, Linear Multilinear Algebra 65 (2017), no. 6, 11711193. MR 3615537

\section{A. Tajmouati}

Sidi Mohamed Ben Abdellah University, Faculty of Sciences Dhar Al Mahraz, Laboratory of Mathematical Analysis and Applications, Fez, Morocco

abdelaziz.tajmouati@usmba.ac.ma

M. Karmouni

Cadi Ayyad University, Multidisciplinary Faculty, Safi, Morocco

mohammed.karmouni@uca.ma

M. B. Mohamed Ahmed

Sidi Mohamed Ben Abdellah University, Faculty of Sciences Dhar Al Mahraz, Laboratory of Mathematical Analysis and Applications, Fez, Morocco

bbaba2012@gmail.com

Received: August 4, 2018

Accepted: April 18, 2019 